bisulphate sequencing ${ }^{10}$ (for profiling DNA methylation), Hi-C ${ }^{11}$ (for capturing chromosome conformation), and chromatin immunoprecipitation $^{6}$ (for detecting histone modifications and, in the longer perspective, transcription factor binding to DNA). Integrating data from these assays, as is routinely done in population-wide analyses, should lead to the most interesting insights. In the most exciting scenario, multiple readouts would be acquired from the same cell, as is already possible for gene expression and DNA methylation ${ }^{12}$. In particular, it would be useful to integrate single-cell ATAC-seq with spatial information from single-cell $\mathrm{Hi}-\mathrm{C}$ to uncover the target genes of cell-specific putative regulatory elements.

Unfortunately, integration of multiple singlecell data sets is challenging because each of the technologies provides only limited coverage of individual cells. The level of coverage is sufficient to classify single cells and discover new subpopulations, but it is much harder to extract information about individual loci. This inflates the real differences between cells and means that even data from identical cells would appear disparate. The problem is exacerbated when integrating multiple sparse single-cell data sets from different assays. Although it may be possible to optimize single-cell assays to recover more information per cell, the next barrier to overcome will be the high sequencing costs of profiling large numbers of single cells at high coverage. Striking a balance between throughput and coverage in single-cell technologies will be an important consideration for the future.

\section{COMPETING FINANCIAL INTERESTS}

The authors declare no competing financial interests.

1. Corces, M.R. et al. Nat. Genet. 48, 1193-1203 (2016).

2. ENCODE Project Consortium. Nature 489, 57-74 (2012).

3. Kundaje, A. et al. Nature 518, 317-330 (2015).

4. Buenrostro, J.D., Giresi, P.G., Zaba, L.C., Chang, H.Y. \& Greenleaf, W.J. Nat. Methods 10, 1213-1218 (2013).

5. Lara-Astiaso, D. et al. Science 345, 943-949 (2014).

6. Rotem, A. et al. Nat. Biotechnol. 33, 1165-1172 (2015).

7. Buenrostro, J.D. et al. Nature 523, 486-490 (2015).

8. Newman, A.M. et al. Nat. Methods 12, 453-457 (2015).

9. Kolodziejczyk, A.A., Kim, J.K., Svensson, V., Marioni, J.C. \& Teichmann, S.A. Mol. Cell 58, 610-620 (2015).

10. Smallwood, S.A. et al. Nat. Methods 11, 817-820 (2014).

11. Nagano, T. et al. Nature 502, 59-64 (2013).

12. Angermueller, C. et al. Nat. Methods 13, 229-232 (2016).

\title{
High-yield chemical synthesis by reprogramming central metabolism
}

\author{
Vivian Y Yu \& Michelle C Y Chang
}

\section{Altering the hardwired stoichiometry of central metabolism in yeast enables efficient synthesis of the isoprenoid $\beta$-farnesene.}

Cellular metabolism comprises a diverse set of reactions that can be engineered to convert a carbon feedstock into a wide range of chemicals ${ }^{1,2}$. Although metabolic engineering approaches to produce fine and commodity chemicals have been devised ${ }^{3}$, it remains difficult to introduce large-scale changes in industrial fermentation hosts, such as Saccharomyces cerevisiae (baker's yeast), so that they can efficiently carry out non-native chemistries that are not necessarily compatible with their existing metabolism ${ }^{4}$. A recent study in Nature from Meadows et al. ${ }^{5}$ at Amyris describes a groundbreaking effort to rewire carbon and energy metabolism in yeast

Vivian Yu \& Michelle Chang are at the Departments of Chemistry and Molecular \& Cell Biology, University of California, Berkeley, USA. Michelle Chang is also at the Chemical Sciences Division, Lawrence Berkeley National Laboratory, Berkeley, USA.

e-mail: mcchang@berkeley.edu in order to produce commercially relevant yields of the isoprenoid $\beta$-farnesene.

All cells share a common set of pathways for carbon assimilation and rearrangement, including glycolysis and the tricarboxylic acid cycle, but different evolutionary pressures have led to species differences in how these reactions are deployed. S. cerevisiae has been bred by brewers and winemakers to produce ethanol from glucose by anaerobic fermentation regardless of the availability of oxygen for respiration ${ }^{6}$. Thus, these yeasts preferentially use glycolysis as the sole energy-producing pathway for generating ATP and NADH, with conversion of pyruvate to ethanol acting as a fermentation pathway to maintain glycolysis (Fig. 1a). As a result, most of the carbon taken up by yeast is retained in glycolysis and fermentation, and very little is shuttled to mitochondria for the tricarboxylic acid cycle and aerobic respiration despite the greater potential yield of ATP from full glucose oxidation ${ }^{6}$. After $\sim 7,000$ years of evolution, this carbon metabolism is hardwired into S. cerevisiae at many levels, which has hampered efforts to divert carbon flux into new products.

Metabolic engineering of $S$. cerevisiae, therefore, presents an interesting challenge for production of the many desirable chemicals that can be made from acetyl coenzyme A (CoA), such as lipids, isoprenoids and polyketides ${ }^{4,7}$. Although carbon is retained in the cytosol where it can be more easily tapped as a building block for downstream pathways, very little of the pyruvate from glycolysis is converted into acetyl-CoA and products derived from it.

Isoprenoids are a large class of natural products that have been long valued for their utility as medicinal agents as well as flavors and fragrances. In earlier generations of the industrial yeast strain used to produce isoprenoids, the native pyruvate dehydrogenase (PDH) bypass pathway was overexpressed in order to generate sufficient cytosolic acetyl-CoA to synthesize the non-native isoprenoids artemisinic acid and farnesene (Fig. 1a) ${ }^{8}$. It took more than 30 optimization steps to construct this strain, which yields $14.3 \mathrm{~g}$ of farnesene per gram of glucose at a rate of $1.26 \mathrm{~g}$ per liter per hour. These metrics are impressive, but the theoretical yield using only central carbon metabolism native to yeast is limited, requiring significantly more glucose (4.76 molecules of glucose per molecule of farnesene or equivalents) and oxygen (7.55 equivalents) for production of farnesene than needed based on the chemical limit (3.5 equivalents glucose and 0 equivalents of oxygen) (Fig. 1a). In particular, the large excess of oxygen precludes efficient production of farnesene because industrial fermentations are constrained by the rate of oxygenation in a fermenter upon scale-up.

To designa moreefficientstrain, Meadows et al. ${ }^{5}$ introduced a set of non-native reactions for acetyl-CoA metabolism to the existing yeast genome-scale metabolic model, which allows pathway stoichiometries to be calculated based on the biochemical reactions available to the host. They found that it was theoretically possible to increase the yield of farnesene from glucose (to 3.81 molecules of glucose needed per molecule of farnesene or equivalents), which is close to the chemical limit, and concomitantly decrease oxygen usage (to 1.85 equivalents) if two different connections to acetyl-CoA were added to the metabolic model.

One new connection diverts carbon flowing through glycolysis at the fructose-6-phosphate stage to the pentose phosphate pathway. This results in production of two-carbon acetylphosphate from the scission of a five-carbon sugar, leaving a three-carbon fragment that is recycled (Fig. 1 $\mathbf{b}$, phosphoketolase) ${ }^{9}$. The acetyl-phosphate can then be converted into acetyl-CoA by phosphotransacetylase. This 

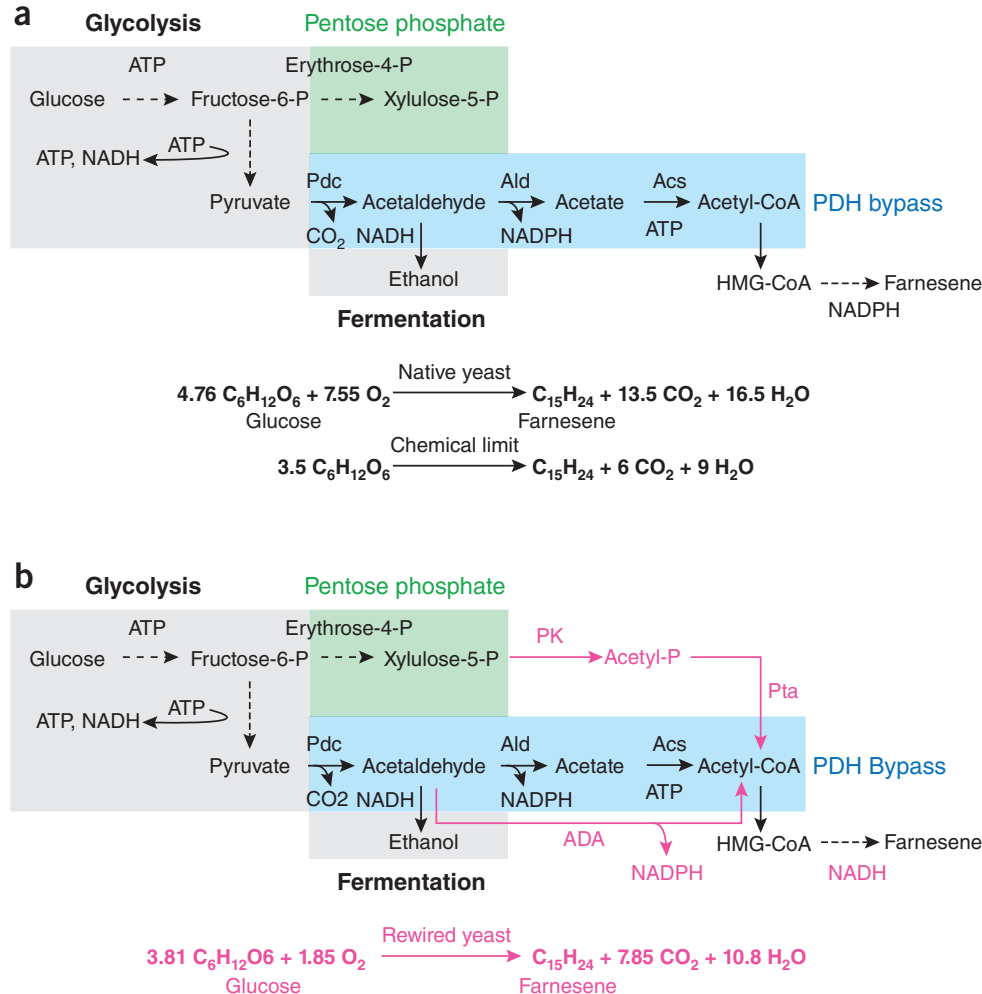

Figure 1 Carbon and oxygen use in yeast central metabolism. (a) Native yeast cannot efficiently produce the isoprenoid farnesene from acetyl-CoA owing to limitations in carbon and oxygen usage. An earlier-generation strain from Amyris uses an overexpressed PDHc bypass (shaded blue) to produce acetyl-CoA along with a NADPH-selective HMG-CoA reductase. (b) The simultaneous introduction of two new pathways in S. cerevisiae to introduce heterologous non-oxidative glycolysis and alternative pyruvate dehydrogenase (PDH) bypass pathways (pink) allows glucose flux to be balanced through these two pathways to achieve an optimal stoichiometry for farnesene production. Reaction stoichiometries are not balanced and the pentose phosphate pathway is simplified for clarity in this schematic. Dashed arrows indicate multiple steps that are not shown. P, phosphate; HMG-CoA, hydroxymethylglutaryl coenzyme A; Pdc, pyruvate decarboxylase, ACS, acetyl-CoA synthetase; PK, phosphoketolase, Pta, phosphotransacetylase; ADA, aldehyde dehydrogenase acylating.

pathway operates without loss of carbon dioxide, thereby increasing the carbon flux into farnesene but without producing the ATP and $\mathrm{NADH}$ needed to synthesize farnesene.

To balance the energetic deficiency caused by this shunt pathway, the authors added an acetaldehyde dehydrogenase (acylating), thereby avoiding the use of ATP in the native pyruvate dehydrogenase $(\mathrm{PDH})$ bypass (Fig. 1b $)^{10}$. As this enzyme generates NADH instead of NADPH, a compensatory cofactor switch was also made in the downstream isoprenoid pathway. Together, these two new connections redistribute glucose flux to balance each other out and reduce carbon loss and production of excess NAD $(\mathrm{P}) \mathrm{H}$, which reduces oxygen demand via aerobic respiration.

These changes were implemented to produce a next-generation isoprenoid strain after identifying candidates for these enzyme activities. Genes encoding phosphoketolase (PK), phosphotransacetylase (Pta) and aldehyde dehydrogenase acylating (ADA) were selected from various organisms and subjected to a genetics screen to choose the ortholog with the best in vivo performance in yeast. An NADH-selective hydroxymethylglutaryl-CoA reductase (HMGr) to replace

\section{Research Highlights} Twitter, @NatureBiotech \#nbtHighlight) sion and activation La Manno, G. et al. Cell 167, 566-580 (2016) receptors

Roybal, K.T. et al. Cell 167, 419-432 (2016) the existing NADPH-selective HMGr in the mevalonate pathway was then found through in vitro biochemical characterization. These four new pathway components were incorporated to generate a new strain that was benchmarked against the previous generation containing only the overexpressed $\mathrm{PDH}$ bypass ${ }^{8}$. A direct comparison showed a $21 \%$ improvement in yield and a $77 \%$ improvement in productivity.

To confirm that these enhancements arose from an alteration in pathway stoichiometry, the authors compared the amounts of glucose and oxygen consumed with the amounts of biomass and farnesene produced. They concluded that the changes made to central metabolism enabled production of farnesene with $25 \%$ less glucose and $75 \%$ less oxygen.

The practical implications of this work are important for industrial fermentation as yields are key drivers of costs and profit margins. The success of the authors' approach also highlights the complexities in engineering central carbon pathways and the role of modeling in optimizing yield and productivity, as a single pathway may not provide the energetic balance required for commercially viable processes.

\section{COMPETING FINANCIAL INTERESTS}

The authors declare no competing financial interests.

1. Bailey, J.E. Science 252, 1668-1675 (1991).

2. Stephanopoulos, G., Aristidou, A.A. \& Nielsen, J. Metabolic Engineering: Principles and Methodologies (Academic Press, Cambridge, MA, 1998).

3. Lee, J.W. et al. Nat. Chem. Biol. 8, 536-546 (2012).

4. Nielsen, J. \& Keasling, J.D. Cell 164, 1185-1197 (2016).

5. Meadows, A.L. et al. Nature 537, 694-697 (2016).

6. Sonnleitner, B. \& Käppeli, O. Biotechnol. Bioeng. 28, 927-937 (1986).

7. van Rossum, H.M., Kozak, B.U., Pronk, J.T. \& van Maris, A.J. Metab. Eng. 36, 99-115 (2016).

8. Paddon, C.J. et al. Nature 496, 528-532 (2013).

9. Bogorad, I.W., Lin, T.-S. \& Liao, J.C. Nature 502 693-697 (2013).

10. Kozak, B.U. et al. Metab. Eng. 21, 46-59 (2014).

Papers from the literature selected by the Nature Biotechnology editors. (Follow us on

Compact and highly active next-generation libraries for CRISPR-mediated gene repres-

Horlbeck, M.A. et al. eLife doi:10.7554/eLife.19760 (2016)

Modular assembly of superstructures from polyphenol-functionalized building blocks Guo, J. et al. Nat. Nanotechnol. doi:10.1038/nnano.2016.172 (2016)

A selective insecticidal protein from Pseudomonas for controlling corn rootworms Schellenberger, U. et al. Science doi:10.1126/science.aaf6056 (2016)

Molecular diversity of midbrain development in mouse, human, and stem cells

Engineering $\mathrm{T}$ cells with customized therapeutic response programs using synthetic notch 